\title{
Reconstruction of generalized impedance functions for 3D acoustic scattering
}

\author{
Olha Ivanyshyn Yaman \\ Department of Mathematics, Izmir Institute of Technology, 35430 Urla, Izmir, Turkey
}

\section{A R T I C L E I N F O}

\section{Article history:}

Received 12 October 2018

Received in revised form 24 April 2019

Accepted 26 April 2019

Available online 3 May 2019

\section{Keywords:}

Inverse problem

Boundary integral equations

Generalized impedance boundary condition

Helmholtz equation

\begin{abstract}
A B S T R A C T
We consider the inverse obstacle scattering problem of determining both of the surface impedance functions from far field measurements for a few incident plane waves at a fixed frequency. The reconstruction algorithm we propose is based on an iteratively regularized Newton-type method and nonlinear integral equations. The mathematical foundation of the method is presented and the feasibility is illustrated by numerical examples.
\end{abstract}

(C) 2019 Elsevier Inc. All rights reserved.

\section{Introduction}

In this study, we are interested in the numerical reconstruction of surface impedance functions from the knowledge of far field measurements of a scattered wave associated with a few incident plane waves at a given frequency. The generalized impedance boundary conditions (GIBC's) are used to model obstacles coated with a thin layer of a penetrable material, obstacles with corrugated surfaces or to model more accurately imperfectly conducting obstacles. These boundary conditions were introduced in 1940s for modeling electromagnetic wave propagation over irregular terrains, [22]. Since then GIBC's are used for simplifying the analytical solutions or reducing the cost of numerical solutions for problems involving complex structures not only in electromagnetics [10] but also in many other disciplines, in particular, three-dimensional acoustic problems, $[1,2,12,16]$.

The inverse problem we are interested in is to determine the surface impedance functions for a given obstacle from the knowledge of the far field pattern for a few incident plane waves. This problem appears in practical application such as NDT for detecting porosity, reconstruction of surface roughness or coating thickness, [3], for modeling related to stealth technology or antennas, [4]. Additionally, it is motivated by the need to minimize the wave reflected by the obstacle in some directions what can be achieved by introducing a coating on the surface of the obstacle. Moreover, the study on this inverse problem lays down the foundation to a reconstruction algorithm for a coated obstacle, i.e. simultaneous recovery of surface impedances and shape of the obstacle.

The literature overview for the inverse GIBC related problems in two dimensions reveals the following research results. In the case of Leontovich boundary condition the problem for reconstruction the impedance and the shape of the obstacle is well-studied, see e.g. [20]. The numerical solution for the general case of a second order impedance boundary condition was investigated by Bourgeois et al. [4,6] with the aid of variational formulation for the solution of the direct problem and by Kress [19] with the solution method completely based on the boundary integral equations. Assuming that the boundary $\Gamma$

E-mail address: olhaivanyshyn@iyte.edu.tr. 
is also unknown the variational approach was extended to the simultaneous reconstruction of the shape and the impedance functions, [5]. A closely related inverse scattering problems with generalized oblique derivative boundary condition was considered in [23] and the linear sampling method was developed for the shape reconstruction, [24].

Regarding the inverse GIBC related problems in three dimensions the currently available literature is scarce. For the inverse impedance problem a nonlinear boundary integral equation approach was proposed in [14] for the case of Leontovich boundary condition. Furthermore, the theoretical study on the boundary integral equation methods for the direct scattering problem with generalized impedance boundary condition was recently undertaken by Kress, [18].

In this paper, we present a reconstruction algorithm based on an iteratively regularized Newton-type method and nonlinear boundary integral equations. More specifically, extending the study of [14] to the second order boundary condition we employ an analogue of the Huygens' principle for the generalized impedance scattering and derive a system of nonlinear boundary integral equations equivalent to the inverse problem.

The paper is organized as follows. Section 2 is devoted to the solution of the direct boundary value problem and to the review on the uniqueness results for the inverse problem. We proceed in Section 3 with reduction of the inverse impedance problem to the system of nonlinear boundary equation. Section 4 is allocated to the description of the iterative algorithm. In Section 5, the numerical implementation of the method is described. Finally, in Section 6 the feasibility of the method is illustrated by numerical examples.

\section{Direct and inverse problem}

To generate synthetic data for the inverse problem we firstly review the known results for the direct problem. Mathematically, the direct scattering problem for an obstacle with generalized impedance boundary condition can be stated as follows. Let $D \subset \mathbb{R}^{3}$ be a simply connected bounded domain with boundary $\Gamma$. Given the incident plane wave $u^{i}(x)=e^{i k x \cdot d}$ with wave number $k>0$ and the direction of propagation $d$ the scattering problem consists in finding the total field $u=u^{s}+u^{i}$ such that $u$ satisfies the Helmholtz equation

$$
\Delta u+k^{2} u=0 \text { in } \mathbb{R}^{3} \backslash \bar{D}
$$

the generalized impedance boundary condition (GIBC)

$$
\frac{\partial u}{\partial v}+i k(\lambda-\operatorname{Div} \mu \text { Grad }) u=0, \quad \text { on } \Gamma
$$

where Grad and Div are surface gradient and surface divergence operators on $\Gamma$ and $\nu$ is the outward unit normal vector to $\Gamma$. For brevity of notations, we introduce the differential operator

$$
\mathcal{G}(\lambda, \mu ; u)=i k(\lambda-\operatorname{Div} \mu \operatorname{Grad}) u
$$

The scattered field has also to satisfy the Sommerfeld radiation condition

$$
\lim _{r \rightarrow \infty} r\left(\frac{\partial u^{s}}{\partial r}-i k u^{s}\right)=0, \quad r=|x|,
$$

uniformly with respect to all directions. The Sommerfeld radiation condition guarantees the following asymptotic behavior of the scattered field

$$
u^{S}(x)=\frac{e^{i k|x|}}{|x|}\left\{u_{\infty}\left(\frac{x}{|x|}\right)+O\left(\frac{1}{|x|}\right)\right\}, \quad|x| \rightarrow \infty
$$

uniformly in all directions with the far field pattern $u_{\infty}$ defined on the unit sphere $\mathbb{S}^{2}$, [8].

Due to [18] we have the following well-posedness for the boundary value problem (2.1)-(2.3).

Theorem 2.1. Let $D \subset \mathbb{R}^{3}$ be a bounded domain with a connected boundary $\Gamma$ of Hölder class $C^{4, \alpha}$. Assume $\lambda \in C^{1}(\Gamma), \mu \in C^{2}(\Gamma)$ with $\operatorname{Re} \lambda, \operatorname{Re} \mu \geq 0$ and $|\mu|>0$. Then there exists a unique solution $u \in H_{\text {loc }}^{2}\left(\mathbb{R}^{3} \backslash \bar{D}\right)$ to (2.1)-(2.3).

Proof. For the sake of completeness, we recall the ideas of proof presented in $[18,19]$. Since $\left.u\right|_{\Gamma} \in H^{\frac{3}{2}}(\Gamma)$ GIBC has to be understood in the weak sense

$$
\int_{\Gamma}\left(\eta \frac{\partial u}{\partial v}+i k \lambda \eta u+i k \eta \operatorname{Grad} \eta \cdot \operatorname{Grad} u\right) d s=0, \quad \forall \eta \in H^{\frac{3}{2}}(\Gamma) .
$$

By Rellich's lemma, [8, Theorem 2.13], the direct problem has at most one solution provided $\operatorname{Re} \lambda, \operatorname{Re} \mu \geq 0$. 
Representing the solution as the combined layer potential

$$
u^{s}(x)=\int_{\Gamma}\left\{\Phi(x, y)+i \frac{\partial \Phi(x, y)}{\partial \nu(y)}\right\} \varphi(y) d s(y), x \in \mathbb{R}^{3} \backslash \bar{D}, \varphi \in H^{\frac{3}{2}}(\Gamma)
$$

substituting it into GIBC (2.2) and using the jump relations of layer potentials yields equivalence of (2.1)-(2.3) to the integrodifferential equation

$$
\varphi-K^{\prime} \varphi-i T \varphi-\mathcal{G}(\lambda, \mu ; S \varphi+i \varphi+i K \varphi)=\left.2 \frac{\partial u^{i}}{\partial v}\right|_{\Gamma}+2 \mathcal{G}\left(\lambda, \mu ;\left.u^{i}\right|_{\Gamma}\right),
$$

where $S$ and $K$ are the single- and double-layer potential operators, correspondingly are defined by

$$
(S \varphi)(x):=2 \int_{\Gamma} \Phi(x, y) \varphi(y) d s(y),(K \varphi)(x):=2 \int_{\Gamma} \frac{\partial \Phi(x, y)}{\partial v(y)} \varphi(y) d s(y),
$$

and $K^{\prime} \varphi:=\frac{\partial S \varphi}{\partial v}, T \varphi:=\frac{\partial K \varphi}{\partial v}$ are their normal derivatives. The operator

$$
\left(K^{\prime} \varphi\right)(x):=2 \int_{\Gamma} \frac{\partial \Phi(x, y)}{\partial v(x)} \varphi(y) d s(y)
$$

is adjoint to the operator $K$ with respect to $L^{2}$ bilinear form. The statement is completed by showing that the modified Laplace-Beltrami operator $\varphi \mapsto-\operatorname{Div} \operatorname{Grad} \varphi+\varphi$ is an isomorphism from $H^{\frac{3}{2}}(\Gamma)$ onto $H^{-\frac{1}{2}}(\Gamma)$, employing boundedness of $S, K: H^{\frac{3}{2}}(\Gamma) \rightarrow H^{\frac{5}{2}}(\Gamma)$ in the case $\Gamma$ being of Hölder class $C^{4, \alpha}$, [17], using compact embedding $I_{H^{\frac{1}{2}}(\Gamma) \hookrightarrow H^{-\frac{1}{2}}(\Gamma)}$ and finally applying the Riesz theory.

Newly the following existence result was proved under the weaker conditions on the boundary and the surface impedance functions, [9].

Theorem 2.2. Let $D \subset \mathbb{R}^{3}$ be a bounded domain with a connected boundary $\Gamma$ of Hölder class $C^{3, \alpha}$. Assume $\lambda \in C(\Gamma)$, $\mu \in C^{1}(\Gamma)$ with $\operatorname{Re} \lambda, \operatorname{Re} \mu \geq 0$ and $|\mu|>0$. Then there exists a unique solution $u \in H_{\mathrm{loc}}^{1,1}\left(\mathbb{R}^{3} \backslash \bar{D}\right)=\left\{u \in H_{\mathrm{loc}}^{1}\left(\mathbb{R}^{3} \backslash \bar{D}\right):\left.u\right|_{\Gamma} \in H^{1}(\Gamma)\right\}$ to $(2.1)-(2.3)$.

We seek the scattered field $u^{s} \in H_{\text {loc }}^{2}\left(\mathbb{R}^{3} \backslash \bar{D}\right)$ in the form of a single-layer potential

$$
u^{s}(x)=\int_{\Gamma} \Phi(x, y) \varphi(y) d s(y), \quad x \in \mathbb{R}^{3} \backslash \bar{D},
$$

where $\Phi(x, y)=\frac{1}{4 \pi} \frac{e^{i k|x-y|}}{|x-y|}, x \neq y$ is the fundamental solution to the Helmholtz equation in $\mathbb{R}^{3}$ and $\varphi \in H^{\frac{1}{2}}(\Gamma)$. Substituting the total field to the boundary condition (2.2) and using the jump relations for the single-layer potential, [8], we obtain the following integro-differential equation

$$
\varphi-K^{\prime} \varphi-\mathcal{G}(\lambda, \mu ; S \varphi)=\left.2 \frac{\partial u^{i}}{\partial v}\right|_{\Gamma}+2 \mathcal{G}\left(\lambda, \mu ;\left.u^{i}\right|_{\Gamma}\right)
$$

It is shown, [18], that the operator $A(\lambda, \mu ; \cdot): H^{\frac{1}{2}}(\Gamma) \rightarrow H^{-\frac{1}{2}}(\Gamma)$ defined by

$$
A(\lambda, \mu ; \varphi):=\varphi-K^{\prime} \varphi-\mathcal{G}(\lambda, \mu ; S \varphi)
$$

has a bounded inverse provided $k^{2}$ is not a Dirichlet eigenvalue for the negative Laplacian in $D$. Hence, our synthetic data $u_{\infty} \in L^{2}\left(\mathbb{S}^{2}\right)$ can be found as follows

$$
u_{\infty}(\hat{x})=\frac{1}{4 \pi} \int_{\Gamma} e^{-i k \hat{x} \cdot y} \varphi(y) d s(y), \quad \hat{x}:=\frac{x}{|x|} \in \mathbb{S}^{2} .
$$

The inverse problem $(I P)$ we are concerned with is formulated as follows.

Given the shape $\Gamma$, the location of the obstacle $D$ and the far field pattern $u_{\infty}$ for several incident plane waves determine the surface impedance functions $\lambda$ and $\mu$.

Before starting to solve the inverse problem we need to figure out what is the minimal number of far field patterns to guarantee the uniqueness of the solution. Due to the result of Bourgeois, Chaulet, Haddar [5] it is known that both 
the shape and the impedance functions are uniquely determined by the far field patterns for an infinite number of incident waves with distinct incident directions and one fixed wave number. The more suitable result for the numerical methods was recently found by Cakoni and Kress, [7,19], in the case of two dimensions. They have shown that three far field patterns corresponding to the scattering of plane waves with different incident directions uniquely determine the impedance function for a given shape $\Gamma$. Unfortunately, there is no straightforward conclusion for the uniqueness in three dimensions. Moreover, extending the counter example given in [19], we can show non-uniqueness for the inverse impedance problem with finite number of far field patterns corresponding to incident spherical wave.

Let $D$ be a ball of radius $R$ centered at the origin and let $\lambda, \mu$ be constants such that $\operatorname{Re} \lambda \geq 0, \operatorname{Re} \mu \geq 0,|\mu|>0$. We consider incident spherical waves $u^{i}(x)=j_{n}(k|x|) Y_{n \ell}(\hat{x}),|\ell| \leq n, n \in \mathbb{N}$ where $j_{n}$ is the spherical Bessel function and $Y_{n \ell}$ is the spherical harmonic of $n$ degree and $\ell$ th order. The corresponding total fields are given by

$$
u_{\ell}(x)=\left(j_{n}(k|x|)-a_{n} h_{n}^{(1)}(k|x|)\right) Y_{n \ell}(\hat{x})
$$

Substituting the total fields in the generalized impedance boundary condition (2.2) and recalling that the spherical harmonics are eigenfunctions of the Laplace-Beltrami operator we find the coefficients

$$
a_{n}=\frac{k j_{n}^{\prime}(k R)+i k\left(\lambda+n(n+1) \mu / R^{2}\right) j_{n}(k R)}{k h_{n}^{(1) \prime}(k R)+i k\left(\lambda+n(n+1) \mu / R^{2}\right) h_{n}^{(1)}(k R)} .
$$

The denominator in (2.9) does not vanish due to Rellich's lemma and the assumptions on the impedance coefficients. Hence, we can choose different combinations of impedances $\lambda$ and $\mu$ giving the same value of $a_{n}$ and $2 n+1$ linear independent total fields.

Note, this model example demonstrates also another difficulties of the inverse impedance problem such as nonlinearity and severe ill-posedness which will be addressed in the following sections.

\section{Numerical solution of the inverse problem}

The main idea of the method we propose is to replace the solution of the inverse problem (IP) by the solution of a system of nonlinear integral equation. To derive the system we employ Green's formula, [8, Theorem 2.5], to the scattered field

$$
u^{s}(x)=\int_{\Gamma}\left\{u^{s}(y) \frac{\partial \Phi(x, y)}{\partial v(y)}-\frac{\partial u^{s}(y)}{\partial v(y)} \Phi(x, y)\right\} d s(y), \quad x \in \mathbb{R}^{3} \backslash \bar{D}
$$

and Green's theorem to the entire solution $u^{i}$ and $\Phi(x, \cdot)$

$$
0=\int_{\Gamma}\left\{u^{i}(y) \frac{\partial \Phi(x, y)}{\partial v(y)}-\frac{\partial u^{i}(y)}{\partial v(y)} \Phi(x, y)\right\} d s(y), \quad x \in \mathbb{R}^{3} \backslash \bar{D} .
$$

Substituting the total field to the boundary condition (2.2) we find its representation in terms of the boundary traces, i.e., for $x \in \mathbb{R}^{3} \backslash \bar{D}$

$$
u(x)=u^{i}(x)+\int_{\Gamma}\left\{\frac{\partial \Phi(x, y)}{\partial v(y)} u(y)+\Phi(x, y) \mathcal{G}(\lambda, \mu ; u)(y)\right\} d s(y)
$$

Considering that the influence of a given object on an incident field is described by a distribution of the so-called "secondary sources" along the surface we may interpret (3.1) as Huygens' principle, [8], for generalized impedance scattering.

As the next step we recall the single- and double-layer operators $S, K: H^{-\frac{1}{2}}(\Gamma) \rightarrow H^{\frac{1}{2}}(\Gamma)$ defined by (2.5) and introduce the far fields for single- and double-layer operators $S_{\infty}, K_{\infty}: H^{-\frac{1}{2}}(\Gamma) \rightarrow L^{2}\left(\mathbb{S}^{2}\right)$ defined by

$$
\begin{aligned}
& \left(S_{\infty} \varphi\right)(\hat{x}):=\frac{2}{4 \pi} \int_{\Gamma} e^{-i k \hat{x} \cdot y} \varphi(y) d s(y), \\
& \left(K_{\infty} \varphi\right)(\hat{x}):=\frac{-2 i k}{4 \pi} \int_{\Gamma} e^{-i k \hat{x} \cdot y} \hat{x} \cdot v(y) \varphi(y) d s(y) .
\end{aligned}
$$

From the jump relations for single- and double-layer potentials, the asymptotic behavior of the Hankel functions we derive the following theorem which lays a foundation for the inversion method. 
Theorem 3.1. For a given boundary $\Gamma$, an incident field $u^{i}$ and the corresponding far field pattern $u_{\infty}$, assume that the surface impedance functions $\lambda, \mu$ and the density $\varphi$ satisfy the system of nonlinear boundary integral equations

$$
\begin{aligned}
& \varphi-K \varphi-S \mathcal{G}(\lambda, \mu ; \varphi)=\left.2 u^{i}\right|_{\Gamma}, \\
& K_{\infty} \varphi+S_{\infty} \mathcal{G}(\lambda, \mu ; \varphi)=u_{\infty} .
\end{aligned}
$$

Then $\lambda, \mu$ are the solutions to the inverse impedance problem (IP).

Proof. Consider the total field expressed via (3.1). Then its far field $u_{\infty}$ is represented by $K_{\infty} \varphi+S_{\infty} \mathcal{G}(\lambda, \mu ; \varphi)$ and hence the data equation (3.3) guarantees that the scattered field has a correct far field pattern. Recalling that $\varphi=\left.u\right|_{\Gamma}$ and taking the Dirichlet trace of (3.1) by the jump relation for layer potentials, [8], we find

$$
2 \varphi=\left.2 u^{i}\right|_{\Gamma}+\varphi+K \varphi+S \mathcal{G}(\lambda, \mu ; \varphi) .
$$

Hence the field equation (3.2) ensures the boundary condition (2.2).

Note, the obtained system is ill-posed due to the data equation which contains the compact operators with exponentially decreasing singular values. We list several methods to solve the system (3.2)-(3.3).

1. Introducing the new unknown $\chi=\mathcal{G}(\lambda, \mu ; \varphi)$ we can interpret the system as linear which can be solved by the Tikhonov regularization. The unknown impedance functions are then solved from the above differential equation.

2. Solving the density $\varphi$ from the field equation and linearizing the data equation.

3. Reversing the order of the equations in the 2 nd method.

4. Simultaneous linearization of both equations with respect to all unknowns.

The first method resembles in some way the direct approach for the inverse problem with the Leontovich boundary condition, [14]. The overview of the methods $2-4$ for the boundary shape reconstruction can be found for example in [9, Section 5], [15]. Since the first method was found to be less stable as compare to the method based on the simultaneous linearization we continue the study on the latter one, method 4.

In order to investigate the properties of the boundary integral operators appearing in (3.2)-(3.3) we introduce a bounded linear operator $A^{\prime}(\lambda, \mu ; \cdot): H^{-1 / 2}(\Gamma) \rightarrow H^{1 / 2}(\Gamma)$ defined by

$$
A^{\prime}(\lambda, \mu ; \varphi):=\varphi-K \varphi-S \mathcal{G}(\lambda, \mu ; \varphi)
$$

and a bounded linear operator $A_{\infty}^{\prime}(\lambda, \mu ; \cdot): H^{-1 / 2}(\Gamma) \rightarrow L^{2}\left(\mathbb{S}^{2}\right)$ defined by

$$
A_{\infty}^{\prime}(\lambda, \mu ; \varphi):=K_{\infty} \varphi+S_{\infty} \mathcal{G}(\lambda, \mu ; \varphi) .
$$

Note that the operator $A^{\prime}(\lambda, \mu ; \cdot)$ is adjoint of the operator $A(\lambda, \mu ; \cdot)$ defined by $(2.8)$ in the dual system $<H^{1 / 2}(\Gamma)$, $H^{-1 / 2}(\Gamma)>$ with respect to $L^{2}$ bilinear form. Applying the Fredholm alternative we find that the operator $A^{\prime}(\lambda, \mu ; \cdot)$ : $H^{-1 / 2}(\Gamma) \rightarrow H^{1 / 2}(\Gamma)$ has a bounded inverse.

Having found the Fréchet derivatives of the operators in (3.2)-(3.3) we are ready to formulate the fully linearized system. The inverse problem is not uniquely solvable for one incident plane wave and uniqueness for the finite number of incident direction is an open problem in three dimensions. Motivated by the uniqueness result in two dimensions we consider the nonlinear system (3.2)-(3.3) for $p \geq 3$ incident plane waves

$$
u_{\ell}^{i}(x)=e^{i k x \cdot d_{\ell}}, d_{\ell} \in \mathbb{S}^{2} .
$$

Given the current approximation $\varphi_{\ell}=\left.u_{\ell}\right|_{\Gamma}, \ell=\overline{1, p}, \lambda, \mu$ the fully linearized system reads

$$
\begin{array}{r}
A^{\prime}\left(\lambda, \mu ; \psi_{\ell}\right)-S \mathcal{G}\left(\eta, \zeta ; \varphi_{\ell}\right)=\left.2 u_{\ell}^{i}\right|_{\Gamma}-A^{\prime}\left(\lambda, \mu ; \varphi_{\ell}\right), 1 \leq \ell \leq p, \\
A_{\infty}^{\prime}\left(\lambda, \mu ; \psi_{\ell}\right)+S_{\infty} \mathcal{G}\left(\eta, \zeta ; \varphi_{\ell}\right)=u_{\infty, \ell}-A_{\infty}^{\prime}\left(\lambda, \mu ; \varphi_{\ell}\right), 1 \leq \ell \leq p,
\end{array}
$$

for the unknown updates $\psi_{\ell}, \eta, \zeta$ of the functions $\varphi_{\ell}, \lambda, \mu$, correspondingly.

In order to apply the Tikhonov regularization we need to investigate injectivity of the system (3.4)-(3.5) at the exact solution. Unfortunately, this issue is not resolved yet since it is directly related to the question of unique reconstruction of the surface impedances. To demonstrate the connection between the uniqueness issue and the injectivity of the operator in the system (3.4)-(3.5) we define a function $V_{\ell}$ for $x \in \mathbb{R}^{3} \backslash \bar{D}$ by

$$
V_{\ell}(x)=2 \int_{\Gamma}\left\{\frac{\partial \Phi(x, y)}{\partial v(y)} \psi_{\ell}(y)+\Phi(x, y) \mathcal{G}\left(\lambda, \mu ; \psi_{\ell}\right)(y)+\Phi(x, y) \mathcal{G}\left(\eta, \zeta ; \varphi_{\ell}\right)(y)\right\} d s(y),
$$


where $\varphi_{\ell}=\left.u_{\ell}\right|_{\Gamma}$ is the restriction of the total field corresponding to the incident plane wave $u_{\ell}^{i}$ to the boundary $\Gamma$. The equation (3.5) guarantees $V_{\infty, \ell}=0$, and by Rellich's lemma we obtain $V_{\ell}=0$ in $\mathbb{R}^{3} \backslash \bar{D}$. From the jump relations for singleand double-layer potentials, [8], it follows that $\psi_{\ell} \equiv 0$. From (3.5) and the assumption $k^{2}$ is not a Dirichlet eigenvalue for the negative Laplacian in $D$ we can conclude that $\mathcal{G}\left(\eta, \zeta ; \varphi_{\ell}\right)=0$, i.e.

$$
(\eta-\operatorname{Div} \zeta \operatorname{Grad}) \varphi_{\ell}=0
$$

This leads us to the unsolved uniqueness problem. Indeed, choosing $D$ to be a ball of radius $R$ centered at the origin, $\eta$ and $\zeta$ to be constants, recalling that the spherical harmonics are dense in $L^{2}\left(\mathbb{S}^{2}\right)$ we arrive to the equation

$$
\eta+n(n+1) \zeta=0 .
$$

Since $\eta$ and $\zeta$ are the unknown updates, we cannot put positivity restriction on their real or imaginary parts and hence there is a nontrivial solution.

The remedy to this might lie in a special choice on incident directions. However, there is no solution available at the moment and this issue will be closed automatically once the uniqueness question is resolved.

\section{Iterative method}

For the numerical solution of the system of boundary integral equations (3.4)-(3.5) we assume that the surface $\Gamma$ is $C^{4, \alpha}$-smooth and homeomorphic to the unit sphere $\mathbb{S}^{2}$, i.e. we introduce a parametrization $z: \mathbb{S}^{2} \rightarrow \Gamma$,

$$
\Gamma:=\left\{z(\hat{x}): \hat{x} \in \mathbb{S}^{2}\right\}
$$

The parametrized single-layer operator $\mathrm{S}: H^{-1 / 2}\left(\mathbb{S}^{2}\right) \rightarrow H^{1 / 2}\left(\mathbb{S}^{2}\right)$ takes the form

$$
(\mathrm{S} v)(\hat{x})=\int_{\mathbb{S}^{2}} \Phi(z(\hat{x}), z(\hat{y})) v(\hat{y}) J_{z}(\hat{y}) d s(\hat{y}), \quad \hat{x} \in \mathbb{S}^{2},
$$

where $v=u \circ z$ and $J_{z}$ is the Jacobian of the transformation. In a similar way we define the parametrized form of the operators $K, S_{\infty}, K_{\infty}$.

Let $\mathrm{D} z$ be the total derivative which maps the tangent plane to $\mathbb{S}^{2}$ to the tangent plane to $\Gamma$. The operator [D $\left.z^{*}\right]^{-1}$ maps the cotangent plane to the unit sphere $\mathbb{S}^{2}$ to the cotangent plane to the given surface $\Gamma$. To define the parametrized form of the operators $\mathcal{G}, A^{\prime}, A_{\infty}^{\prime}$ we use the following transformation formulas

$$
\begin{aligned}
& (\operatorname{Grad} u) \circ z=\left[\mathrm{D}^{*}\right]^{-1} \operatorname{Grad}_{\mathbb{S}^{2}}(u \circ z), \\
& (\operatorname{Div} v) \circ z=\frac{1}{J_{z}} \operatorname{Div}_{\mathbb{S}^{2}}\left(J_{z}[\mathrm{D} z]^{-1}(v \circ z)\right) .
\end{aligned}
$$

The parametrized form of the system (3.4)-(3.5) for a single incident wave reads

$$
F_{\varphi, \lambda, \mu}^{\prime}\left[\begin{array}{l}
\psi \\
\eta \\
\zeta
\end{array}\right]=b-F\left[\begin{array}{l}
\varphi \\
\lambda \\
\mu
\end{array}\right], \quad b=\left[\begin{array}{l}
2 u^{i} \circ z \\
u_{\infty}
\end{array}\right]
$$

with $F, F_{\varphi, \lambda, \mu}^{\prime}: H^{-1 / 2}\left(\mathbb{S}^{2}\right) \times H^{s_{\lambda}}\left(\mathbb{S}^{2}\right) \times H^{s_{\mu}}\left(\mathbb{S}^{2}\right) \rightarrow H^{1 / 2}\left(\mathbb{S}^{2}\right) \times L^{2}\left(\mathbb{S}^{2}\right)$ given by

$$
F\left[\begin{array}{l}
\varphi \\
\lambda \\
\mu
\end{array}\right]=\left[\begin{array}{l}
\mathrm{A}^{\prime}[\lambda, \mu] \varphi \\
\mathrm{A}_{\infty}^{\prime}[\lambda, \mu] \varphi
\end{array}\right] \text { and } F_{\varphi, \lambda, \mu}^{\prime}\left[\begin{array}{l}
\psi \\
\eta \\
\zeta
\end{array}\right]=F\left[\begin{array}{l}
\psi \\
\lambda \\
\mu
\end{array}\right]+\left[\begin{array}{l}
-\mathrm{S} \\
\mathrm{S}_{\infty}
\end{array}\right] \mathrm{G}(\lambda, \mu ; \varphi) .
$$

By a slight abuse of notation, we use the same symbols for the parametrized densities and the impedance functions.

The inverse surface problem cannot be solved with the far field for a single incident field therefore we extend the operator in (4.1) to the case of $p$ incident directions, i.e. let

$$
\boldsymbol{\varphi}=\left[\varphi_{1}, \ldots, \varphi_{p}\right]^{T} \text { and } \quad \boldsymbol{F}\left[\begin{array}{l}
\varphi \\
\lambda \\
\mu
\end{array}\right]=\left[F\left[\begin{array}{l}
\varphi_{1} \\
\lambda \\
\mu
\end{array}\right], \ldots, F\left[\begin{array}{l}
\varphi_{p} \\
\lambda \\
\mu
\end{array}\right]\right]^{T} .
$$

We compute an approximate solution to

$$
\boldsymbol{F}_{\boldsymbol{\varphi}, \lambda, \mu}^{\prime}\left[\begin{array}{l}
\boldsymbol{\psi} \\
\eta \\
\zeta
\end{array}\right]=\boldsymbol{b}-F\left[\begin{array}{l}
\boldsymbol{\varphi} \\
\lambda \\
\mu
\end{array}\right]
$$


by the Iteratively Regularized Gauss-Newton Method (IRGNM), [13]. One iteration of the method can be written as follows

$$
\begin{array}{r}
{\left[\begin{array}{l}
\varphi^{N+1} \\
\lambda^{N+1} \\
\mu^{N+1}
\end{array}\right]:=\underset{\psi, \eta, \zeta}{\operatorname{argmin}}\left(\left\|\sum_{\ell=1}^{p} F_{\varphi_{\ell}^{N}, \lambda^{N}, \mu^{N}}^{\prime}\left[\begin{array}{l}
\psi_{\ell} \\
\eta \\
\zeta
\end{array}\right]-b_{\ell}+F\left[\begin{array}{l}
\varphi_{\ell}^{N} \\
\lambda^{N} \\
\mu^{N}
\end{array}\right]\right\|^{2}\right.} \\
\left.+\alpha_{N} \sum_{\ell=1}^{p}\left\|\psi_{\ell}+\varphi_{\ell}^{N}-\varphi_{\ell}^{0}\right\|^{2}+\beta_{N}\left\|\eta+\lambda^{N}-\lambda^{0}\right\|^{2}+\gamma_{N}\left\|\zeta+\mu^{N}-\mu^{0}\right\|^{2}\right) .
\end{array}
$$

Here the regularization parameters are chosen of the form $\alpha_{N}=\chi^{-N} \alpha_{0}, \beta_{N}=\chi^{-N} \beta_{0}$ and $\gamma_{N}=\chi^{-N} \gamma_{0}$, with $\chi>1$. For a given approximation $(\varphi, \lambda, \mu)$ on the $N$ th iteration the correction $(\boldsymbol{\psi}, \eta, \zeta)$ is found as the unique solution to the following regularized linear equation

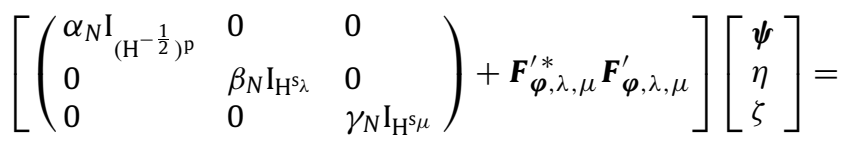

$$
\begin{aligned}
& \boldsymbol{F}_{\boldsymbol{\varphi}, \lambda, \mu}^{\prime *}\left(\boldsymbol{b}-\boldsymbol{F}\left[\begin{array}{c}
\boldsymbol{\varphi} \\
\lambda \\
\mu
\end{array}\right]\right)+\left[\begin{array}{c}
\alpha_{N}\left(\boldsymbol{\varphi}-\boldsymbol{\varphi}^{0}\right) \\
\beta_{N}\left(\lambda-\lambda^{0}\right) \\
\gamma_{N}\left(\mu-\mu^{0}\right)
\end{array}\right] .
\end{aligned}
$$

The $L^{2}$ adjoint to the operator occurring in the final system $F_{\varphi, \lambda, \mu}^{\prime}$ is found as below

$$
F_{\varphi, \lambda, \mu}^{\prime *}\left[\begin{array}{l}
g \\
g_{\infty}
\end{array}\right]=\left[\begin{array}{l}
\mathrm{A}^{\prime}(\lambda, \mu ; g)+\mathrm{K}_{\infty}^{\prime}+\mathrm{G}\left(\lambda, \mu ; \mathrm{S}_{\infty} g_{\infty}\right) \\
-i k \varphi\left(\mathrm{S} g-\mathrm{S}_{\infty}^{*} g_{\infty}\right) \\
i k \operatorname{Div} \varphi\left(\operatorname{Grad} \mathrm{S}_{-}-\mathrm{S}_{\infty}^{*} g_{\infty}\right)
\end{array}\right] .
$$

The iterative scheme.

Step 1. Choose an initial pair of surface impedance functions $(\lambda, \mu)$ and solve the initial densities $\varphi$ from the parametrized form of the well-posed integral equation of the second kind (3.2) for $p$ incident plane waves.

Step 2. Given the approximation $(\varphi, \lambda, \mu)$ solve the system (4.2) for the corrections $\psi, \eta, \zeta$.

Step 3. Update $(\varphi, \lambda, \mu)$ by adding $(\psi, \eta, \zeta)$.

Step 4. Repeat the last two steps until a stopping criterion is fulfilled, e.g. Morozov's discrepancy principle for the noise level $\delta$ and $\tau>1$

$$
\left(\sum_{\ell=1}^{p}\left\|\mathrm{~A}_{\infty}^{\prime}\left(\lambda, \mu ; \varphi_{\ell}\right)-u_{\infty, \ell}\right\|_{L^{2}}\right)^{1 / 2} \leq \tau \delta
$$

\section{Numerical implementation}

To obtain a fully discrete version of (4.2) we apply the fully discrete Galerkin method by Ganesh and Graham, [11], to the parametrized linear boundary integral equations. The method is based on approximations by spherical harmonics and converges super algebraically in the case of smooth boundaries. We start with the numerical integration formula over the unit sphere for a continuous function, the so called Gauss trapezoidal product rule,

$$
\begin{array}{r}
\int_{\mathbb{S}^{2}} u(\hat{x}) d s(\hat{x}) \approx \sum_{\rho=0}^{2 n+1} \sum_{\tau=1}^{n+1} \mu_{\rho} \nu_{\tau} u\left(\hat{x}_{\rho \tau}\right), \quad \hat{x}_{\rho \tau}=\hat{x}\left(\theta_{\tau}, \phi_{\rho}\right), \\
\mu_{\rho}=\frac{\pi}{n+1}, \phi_{\rho}=\frac{\rho \pi}{n+1}, \theta_{\tau}=\arccos \zeta_{\tau},
\end{array}
$$

where $\zeta_{\tau}$ are the zeros of the Legendre polynomial $P_{n+1}^{0}$ of degree $n+1$ and $\nu_{\tau}$ are the corresponding Gauss-Legendre weights. The formula (5.1) is exact for the scalar spherical polynomials of order less than or equal to $2 n+1$. This induces the discrete inner product $(\cdot, \cdot)_{n}$

$$
\left(\varphi_{1}, \varphi_{2}\right)_{n}=\sum_{\rho=0}^{2 n+1} \sum_{\tau=1}^{n+1} \mu_{\rho} \nu_{\tau} \varphi_{1}\left(\hat{x}_{\rho \tau}\right) \overline{\varphi_{2}\left(\hat{x}_{\rho \tau}\right)}
$$

on the space of all scalar spherical harmonics $Y_{l j}$, for $j=-l, \ldots, l$ and $l=1,2, \ldots$, of degree less than or equal to $n$. We introduce a projection operator $\ell_{n}$ defined by 


$$
\ell_{n} u=\sum_{l=0}^{n} \sum_{j=-l}^{l}\left(u, Y_{l j}\right)_{n} Y_{l j}
$$

For the numerical approximation of the integral operators with smooth kernels $\mathrm{K}_{\infty}, \mathrm{S}_{\infty}$, we apply the Gauss trapezoidal product rule. The singularity in the weakly singular kernels of the operators $\mathrm{K}, \mathrm{K}^{\prime}, \mathrm{S}$, is moved by an orthogonal transformation to the north pole $\hat{n}=(0,0,1)^{T}$. To approximate the resulting integrals we use the modified Gauss trapezoidal rule

$$
\int_{\mathbb{S}^{2}} \frac{u(\hat{x})}{|\hat{n}-\hat{x}|} d s(\hat{x}) \approx \int_{\mathbb{S}^{2}} \frac{\left(\ell_{n} u\right)(\hat{x})}{|\hat{n}-\hat{x}|} d s(\hat{x})=\sum_{\rho=0}^{2 n+1} \sum_{\tau=1}^{n+1} \alpha_{\tau} \mu_{\rho} \nu_{\tau} u\left(\hat{x}_{\rho \tau}\right), \alpha_{\tau}=\sum_{l=0}^{n} P_{l}^{0}\left(\zeta_{\tau}\right),
$$

which is based on the fact that the scalar spherical harmonics are eigenfunctions of the single-layer potential on the sphere.

To approximate the surface differential operators we introduce the vectorial spherical harmonics

$$
\mathcal{Y}_{l j}^{(1)}=\frac{1}{\sqrt{l(l+1)}} \operatorname{Grad}_{\mathbb{S}^{2}} Y_{l j}, \quad \mathcal{Y}_{l j}^{(2)}=\frac{1}{\sqrt{l(l+1)}} \operatorname{curl}_{\mathbb{S}^{2}} Y_{l j},
$$

for $j=-l, \ldots, l$ and $l=1,2, \ldots$ which form a complete orthonormal system in the spaces of tangent vectors fields $L_{t}^{2}\left(\mathbb{S}^{2}\right)$. The corresponding projection operator $\mathcal{L}_{n}$ on the space generated by the orthonormal basis of tangential vector spherical harmonics is defined by

$$
\mathcal{L}_{n} v=\sum_{i=1}^{2} \sum_{l=1}^{n} \sum_{j=-l}^{l}\left(v \mid \mathcal{Y}_{l j}^{(i)}\right)_{n} \mathcal{Y}_{l j}^{(i)}
$$

where $\left(v_{1} \mid v_{2}\right)_{n}=\sum_{\rho=0}^{2 n+1} \sum_{\tau=1}^{n+1} \mu_{\rho} v_{\tau} v_{1}\left(\hat{x}_{\rho \tau}\right) \cdot \overline{v_{2}\left(\hat{x}_{\rho \tau}\right)}$.

Both impedance functions are approximated by the scalar spherical harmonics of degree $K$, i.e.

$$
\mu \approx \sum_{l j, l=1}^{K} \mu_{l j} Y_{l j}
$$

The surface differential operator $\mathrm{G}(\lambda, \mu ; \cdot)$ can be approximated as follows

$$
\operatorname{Div} \mu \operatorname{Grad}_{\mathbb{S}^{2}} Y_{l j} \approx \frac{1}{J_{z}} \operatorname{Div}_{\mathbb{S}^{2}} \mathcal{L}_{n} \mu \operatorname{Grad}_{\mathbb{S}^{2}} Y_{l j} .
$$

Since

$$
\operatorname{Div}_{\mathbb{S}^{2}} \operatorname{Grad}_{\mathbb{S}^{2}}=\Delta_{\mathbb{S}^{2}}, \operatorname{Div}_{\mathbb{S}^{2}} \operatorname{Curl}_{\mathbb{S}^{2}}=0 \text {, and } \Delta_{\mathbb{S}^{2}} Y_{l j}=-l(l+1) Y_{l j}
$$

we obtain

$$
\left(\operatorname{Div} \mu \operatorname{Grad}_{\mathbb{S}^{2}} Y_{l j}, Y_{l^{\prime} j^{\prime}}\right) \approx \sum_{p q, p=1}^{n}\left(\frac{1}{J_{z}} Y_{p q}, Y_{l^{\prime} j^{\prime}}\right)_{n}\left(\mu \operatorname{Grad}_{\mathbb{S}^{2}} Y_{l j} \mid \mathcal{Y}_{p q}^{(1)}\right)_{n}(-\sqrt{p(p+1)}) .
$$

For the representation of the tangential gradient of the spherical harmonics we refer to [21]. The fully discrete system (4.2) is solved by the conjugate gradient (CG) method. In the CG algorithm we compute $L^{2}$ adjoint $F_{\varphi, \lambda, \mu}^{\prime *}$ and evaluate norms in corresponding $H^{s}\left(\mathbb{S}^{2}\right)$ spaces for $s \in \mathbb{R}$ which can be characterized by

$$
H^{s}\left(\mathbb{S}^{2}\right)=\left\{v=\sum_{l=0}^{\infty} \sum_{j=-l}^{l} v_{l j} Y_{l j}, v_{l j} \in \mathbb{C}, \sum_{l=0}^{\infty} \sum_{j=-l}^{l}\left(1+l^{2}\right)^{s}\left|v_{l j}\right|^{2}<\infty\right\} .
$$

\section{Numerical experiments}

In this final section, we illustrate the feasibility of the method by several numerical experiments. The synthetic data are obtained by solving the boundary integral equation (2.7) with $n_{s y n}=20$ for an ellipsoid with parametrization

$$
z(\theta, \phi)=(\sin \theta \cos \phi, 0.5 \sin \theta \sin \phi, 0.5 \cos \theta), \quad \theta \in[0, \pi], \varphi \in[0,2 \pi]
$$

and the incident directions $(1,0,0),(0,0,1),(0,0,-1)$ depicted on Fig. 1 . The performance of the method is investigated 

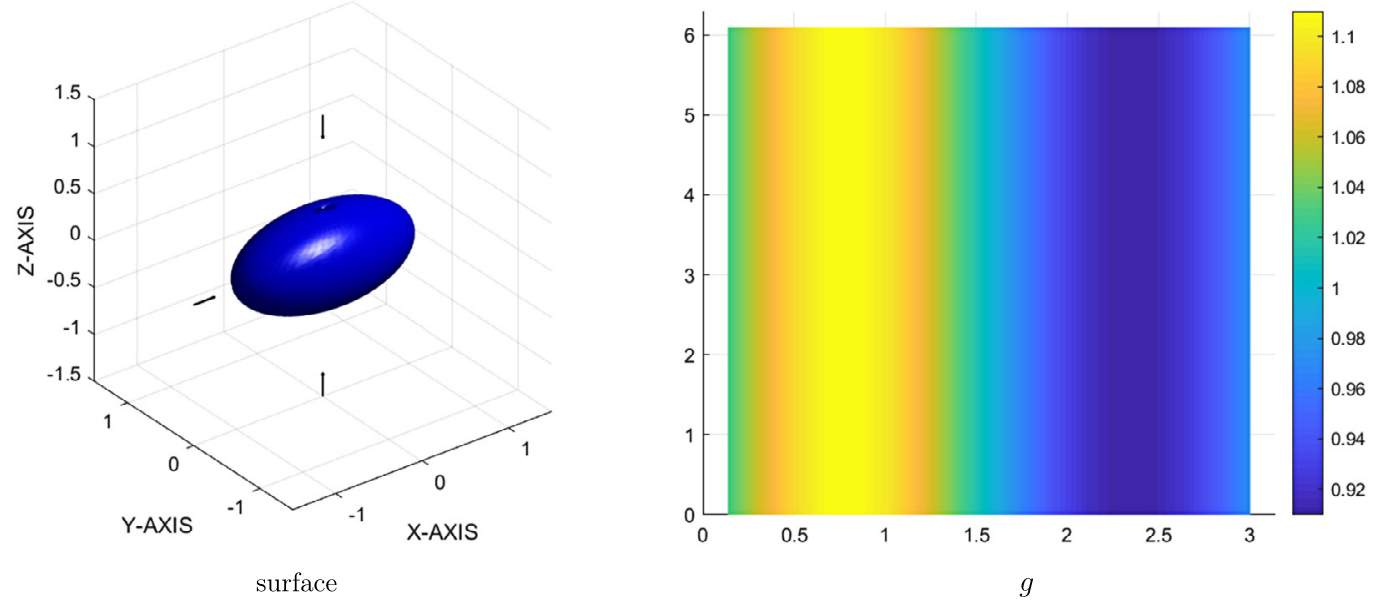

Fig. 1. The scatterer $D$ and the impedance. (For interpretation of the colors in the figure(s), the reader is referred to the web version of this article.)

on the examples where the impedance functions are linear combinations of a constant and the function $g$ given by

$$
g(\theta, \phi)=\frac{1}{1-0.1 \sin 2 \theta}, \quad \theta \in[0, \pi]
$$

The perturbed far field pattern was generated as detailed below

$$
u_{\infty, \ell}^{\delta}=u_{\infty, \ell}+\Theta_{\ell}, \quad \sum_{\ell=1}^{p}\left\|\Theta_{\ell}\right\|_{L^{2}}^{2}<\delta^{2}, \delta=0.02, p=3,
$$

where $\Theta_{\ell}$ is a random complex variable with normally distributed real and imaginary parts. The wave number was chosen $k=\pi / 2$ such that the wavelength $2 \pi / k$ is of a comparable size to the diameter of the obstacle $D$ since we consider scattering for frequencies in the resonance region. The discretization parameters were chosen as follows: $n=15$ and $K=5$. The regularization parameters in (4.2) are selected as $\alpha_{N}=\beta_{N}=\gamma_{N}=\alpha_{0} \chi^{N}, \alpha_{0}=0.001, \chi=10 / 11$. The indexes for the Sobolev space used in the parametrization of impedance functions $\lambda$ and $\mu$ are chosen as $s_{\lambda}=1.1$ and $s_{\mu}=2.1$, correspondingly. The iterations are terminated according to the Morozov's discrepancy principle (4.3) with $\tau=1.001$. The reconstructions are obtained from the far field pattern for 3 incident directions if not stated otherwise.

In the first example we consider the case when the imaginary parts $\operatorname{Im} \lambda, \operatorname{Im} \mu$ of the surface impedance functions are known. The sought surface impedance functions are chosen as follows

$$
\lambda=g, \quad \mu=\lambda .
$$

We test the inversion algorithm for the initial guess $\lambda^{0}=1, \mu^{0}=1$. As can be seen from the Fig. 2a) the impedance function $\lambda$ is accurately reconstructed whereas $\mu$ is affected by the noise more substantially. Increasing the number of incident directions to 6 improves slightly the quality of the reconstruction and reduces the number of iterations, see Fig. 2b). Under the plots we included the relative errors for surface impedance functions, defined by $\operatorname{err}_{\lambda}^{N}:=\left\|\lambda^{N}-\lambda\right\|_{L^{2}} /\|\lambda\|_{L^{2}}$, $\operatorname{err}_{\mu}^{N}:=\left\|\mu^{N}-\mu\right\|_{L^{2}} /\|\mu\|_{L^{2}}$, where $N$ is the iteration number.

In the next example we consider impedances such that $\operatorname{Re} \lambda \geq 0, \operatorname{Im} \lambda \leq 0$ and $\operatorname{Im} \mu<0, \operatorname{Re} \mu \geq 0$, in particular, let

$$
\lambda=g, \quad \mu=-i \bar{\lambda} .
$$

The reason of choosing the above restrictions on the impedance functions is due to the result by Bourgeois and Haddar, [6], which states that if $\operatorname{Im} \lambda \leq 0, \operatorname{Re} \mu \geq 0$ is known and $\mu=$ const then the uniqueness and local stability for the method based on the impedance to far field operator (2D) is guaranteed.

As we can see in Fig. 3, the method proposed in this paper provides accurate reconstructions of both surface impedance functions for only 3 incident plane waves and $\lambda^{0}=1, \mu^{0}=-i$. The quality of the reconstructions of unknown $\operatorname{Im} \lambda, \operatorname{Re} \mu$ has improved significantly as compare to the previous example, Fig. 2a).

Further, we investigate the impact of the $\operatorname{sign}$ of $\operatorname{Im} \mu$ on the quality of the reconstruction for a more general case. Let the functions be chosen in the following way

$$
\lambda=g-0.2 i, \quad \mu=-i \bar{\lambda}, \quad \tilde{\mu}=i \lambda
$$

with the corresponding initial guesses $\lambda^{0}=1, \mu^{0}=-i$, and $\tilde{\mu}^{0}=i$. Similar as observed in the previous example the reconstruction is much more accurate in the case $\operatorname{Im} \mu<0$, see Fig. 4 . 


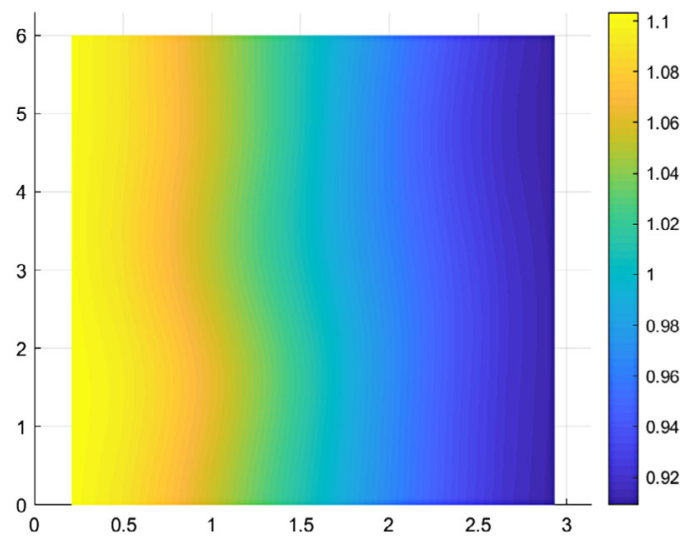

a) $\operatorname{Re} \lambda, \operatorname{err}_{\lambda}^{7}=0.030661$

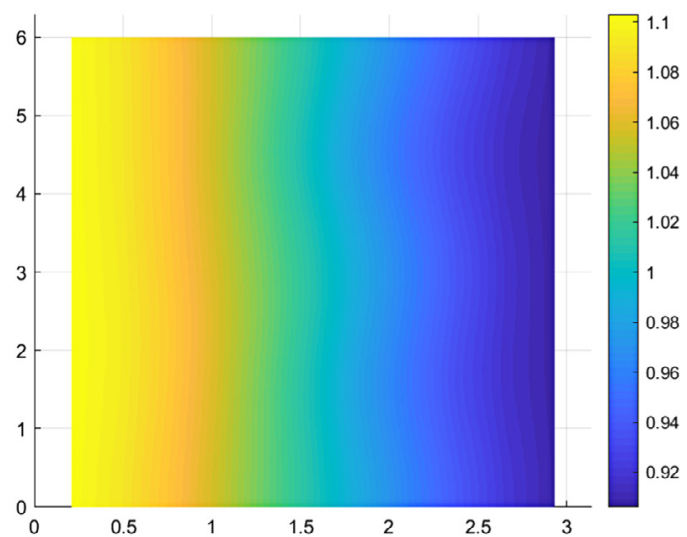

b) $\operatorname{Re} \lambda, \operatorname{err}_{\lambda}^{5}=0.030138$

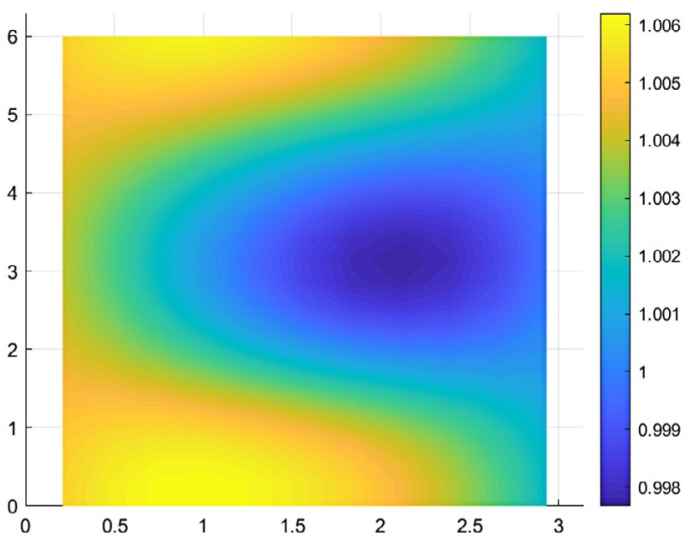

$\operatorname{Re} \mu, \operatorname{err}_{\mu}^{7}=0.072075$

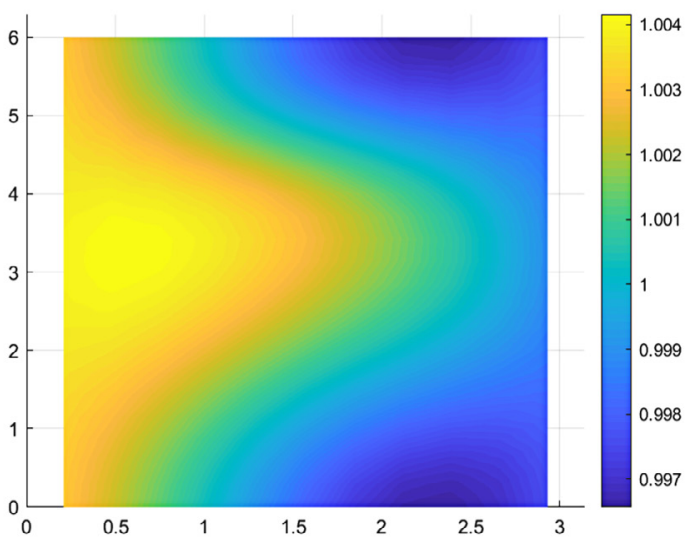

$\operatorname{Re} \mu, \operatorname{err}_{\mu}^{5}=0.071784$

Fig. 2. Reconstruction for $\lambda=g, \mu=\lambda$ with 3 a) and 6 b) incident waves.
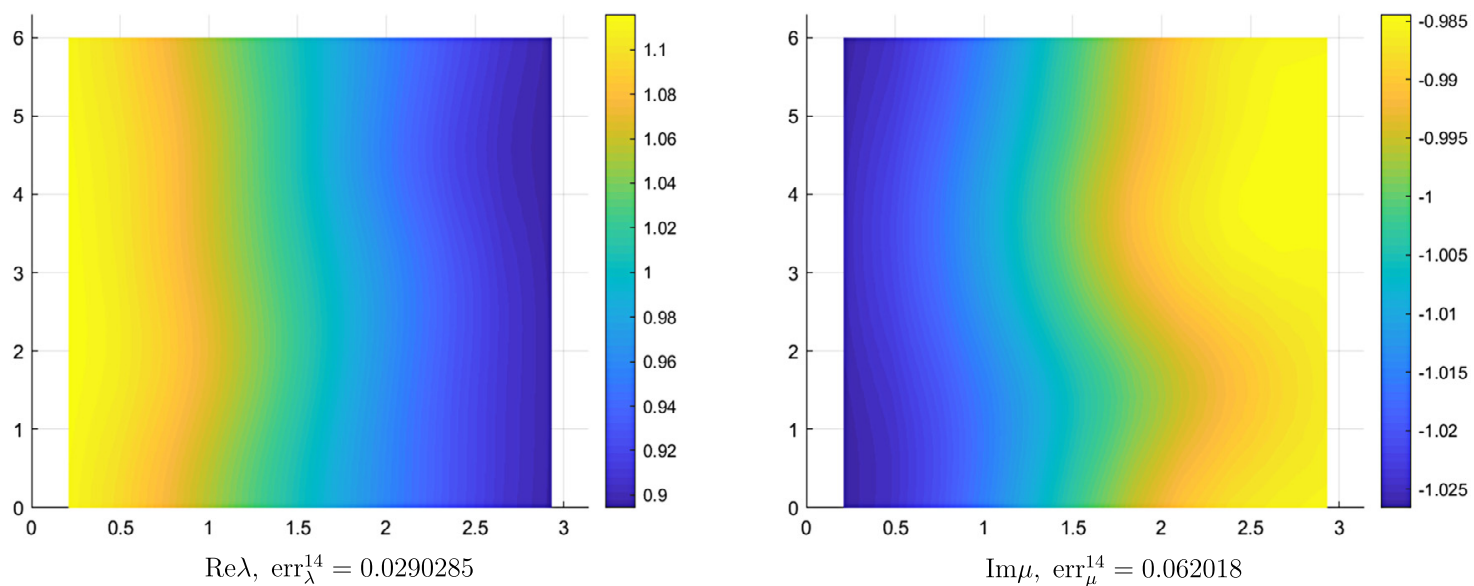

Fig. 3. Reconstruction for $\lambda=g, \mu=-i \bar{\lambda}$.

The last example illustrates the behavior of the algorithm for classical Leontovich boundary condition, i.e. $\mu=0$. The reconstruction of the impedance function $\lambda=g-0.2 i$ for the initial condition $\lambda^{0}=1$ is outlined in Fig. 5. Employing the algorithm twice, i.e. finding the proper initial guess as a constant $(K=0)$ at the first step, improves the quality of reconstructions and allows to use a less accurate initial guess.

To summarize, the proposed inversion method is efficient from the computational point of view since the solutions of boundary value problems appearing in the classical Newton iteration are replaced by matrix-vector products. The algorithm 

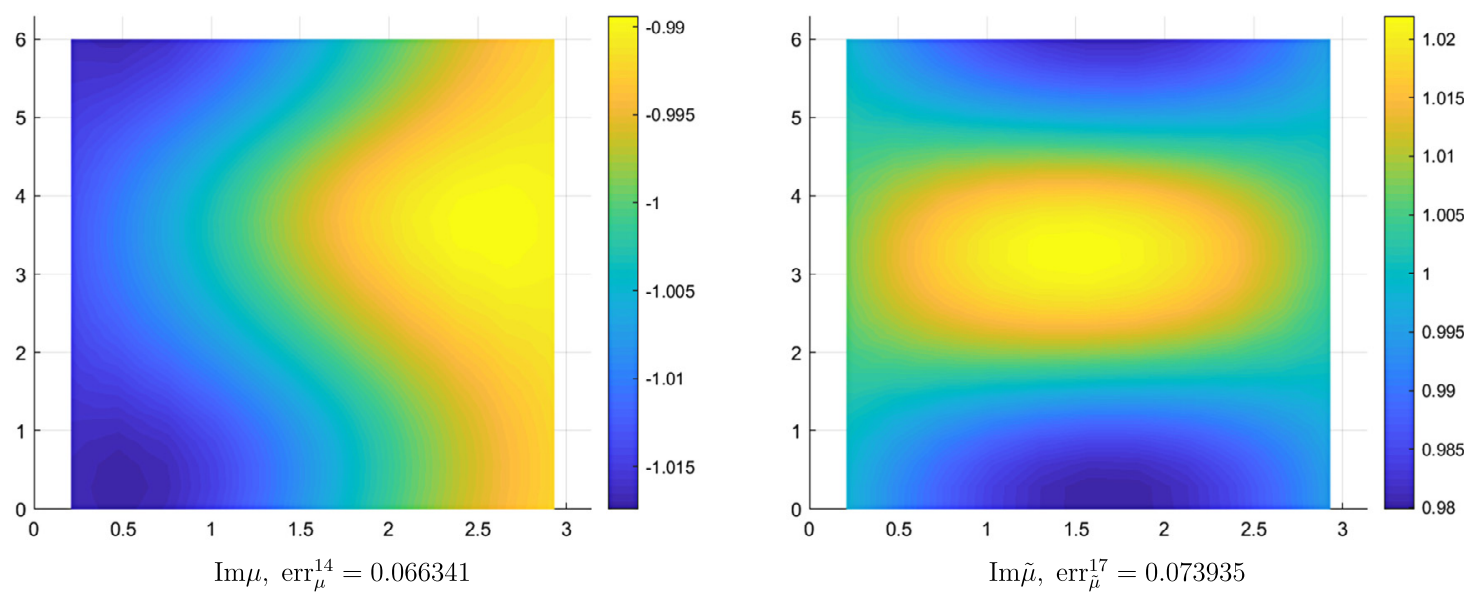

Fig. 4. Reconstruction for $\lambda=g-0.2 i, \mu=-i \bar{\lambda}, \tilde{\mu}=i \lambda$.
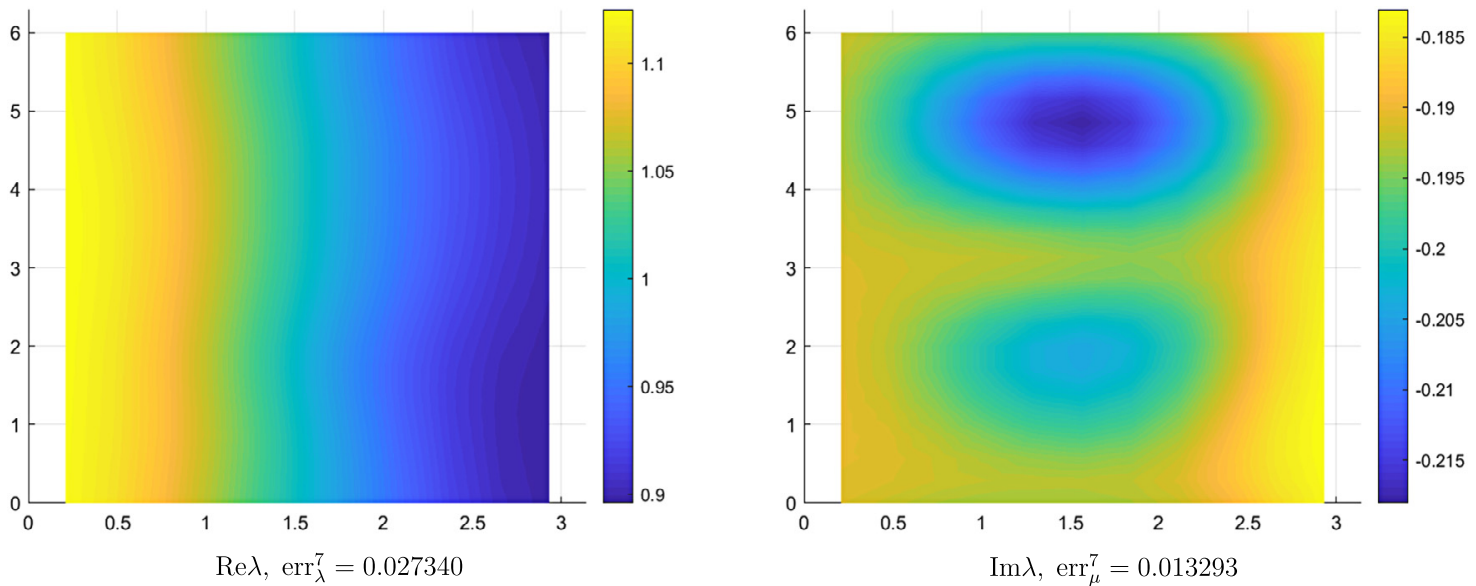

Fig. 5. Reconstruction for $\lambda=g-0.2 i, \mu=0$.

provides accurate reconstructions of the first impedance function $\lambda$ and satisfactory identifications of $\mu$ under some restrictions on the surface impedance functions. In general, in the agreement with results $[4,19]$ for two dimensional case, we note that the simultaneous reconstruction of both impedance function is sensitive to noise, especially the identification of the second impedance function.

\section{Acknowledgement}

The research was supported by the Scientific and Technological Research Council of Turkey (TÜBITAK) through Project No: 116F299. The author thanks F. Le Louër for sharing the code approximating surface derivatives, [16].

\section{References}

[1] X. Antoine, H. Barucq, On the construction of approximate boundary conditions for solving the interior problem of the acoustic scattering transmission problem, in: Domain Decomposition Methods in Science and Engineering, in: Lect. Notes Comput. Sci. Eng., vol. 40, Springer, Berlin, 2005, pp. 133-140.

[2] X. Antoine, H. Barucq, L. Vernhet, High-frequency asymptotic analysis of a dissipative transmission problem resulting in generalized impedance boundary conditions, Asymptot. Anal. 26 (3-4) (2001) 257-283.

[3] B. Aslanyürek, H. Sahintürk, Reconstruction of thickness variation of a dielectric coating through the generalized impedance boundary conditions, ESAIM: Math. Model. Numer. Anal. 48 (4) (2014) 1011-1027.

[4] L. Bourgeois, N. Chaulet, H. Haddar, Stable reconstruction of generalized impedance boundary conditions, Inverse Probl. 27 (9) (2011) 095002 , p. 26.

[5] L. Bourgeois, N. Chaulet, H. Haddar, On simultaneous identification of the shape and generalized impedance boundary condition in obstacle scattering, SIAM J. Sci. Comput. 34 (3) (2012) A1824-A1848.

[6] L. Bourgeois, H. Haddar, Identification of generalized impedance boundary conditions in inverse scattering problems, Inverse Probl. Imaging 4 (1) (2010) 19-38.

[7] F. Cakoni, R. Kress, Integral equation methods for the inverse obstacle problem with generalized impedance boundary condition, Inverse Probl. 29 (1) (2013) 015005, p. 19. 
[8] D. Colton, R. Kress, Inverse Acoustic and Electromagnetic Scattering Theory, third edition, Applied Mathematical Sciences, vol. 93, Springer, New York, 2013.

[9] D. Colton, R. Kress, Inverse Acoustic and Electromagnetic Scattering Theory, fourth edition, Applied Mathematical Sciences, Springer, New York, 2019, to appear.

[10] M. Duruflé, H. Haddar, P. Joly, High order generalized impedance boundary conditions in electromagnetic scattering problems, C. R. Acad. Sci. Paris, Sér. Phys. 7 (2006) 533-542.

[11] M. Ganesh, I.G. Graham, A high-order algorithm for obstacle scattering in three dimensions, J. Comput. Phys. 198 (1) (2004) $211-242$.

[12] H. Haddar, P. Joly, H.-M. Nguyen, Generalized impedance boundary conditions for scattering by strongly absorbing obstacles: the scalar case, Math. Models Methods Appl. Sci. 15 (8) (2005) 1273-1300.

[13] T. Hohage, Logarithmic convergence rates of the iteratively regularized Gauss-Newton method for an inverse potential and an inverse scattering problem, Inverse Probl. 13 (5) (1997) 1279-1299.

[14] O. Ivanyshyn, R. Kress, Inverse scattering for surface impedance from phase-less far field data, J. Comput. Phys. 230 (9) (2011) $3443-3452$.

[15] O. Ivanyshyn, R. Kress, P. Serranho, Huygens' principle and iterative methods in inverse obstacle scattering, Adv. Comput. Math. 33 (4) (2010) 413-429.

[16] D. Kateb, F. Le Louër, Generalized impedance boundary conditions and shape derivatives for 3D Helmholtz problems, Math. Models Methods Appl. Sci. 26 (10) (2016) 1995-2033.

[17] A. Kirsch, Surface gradients and continuity properties for some integral operators in classical scattering theory, Math. Methods Appl. Sci. 11 (6) (1989) 789-804.

[18] R. Kress, Some old and some new results in inverse obstacle scattering, in: U. Langer, D. Pauly, S.I. Repin (Eds.), Maxwell's Equations: Analysis and Numerics. Radon Series on Computational and Applied Mathematics, 2016.

[19] R. Kress, Integral equation methods in inverse obstacle scattering with a generalized impedance boundary condition, in: Contemporary Computational Mathematics-A Celebration of the 80th Birthday of Ian Sloan, Springer, 2018, pp. 721-740.

[20] J. Liu, G. Nakamura, M. Sini, Reconstruction of the shape and surface impedance from acoustic scattering data for an arbitrary cylinder, SIAM J. Appl. Math. 67 (4) (2007) 1124-1146.

[21] F. Le Louër, A spectrally accurate method for the direct and inverse scattering problems by multiple 3D dielectric obstacles, ANZIAM J. 59 (E) (Feb. 2018) E1-E49.

[22] T.B. Senior, J.L. Volakis, Approximate Boundary Conditions in Electromagnetics, Number 41, IET, 1995.

[23] H. Wang, J. Liu, The two-dimensional direct and inverse scattering problems with generalized oblique derivative boundary condition, SIAM J. Appl. Math. 75 (2) (2015) 313-334.

[24] H. Wang, J. Liu, An inverse scattering problem with generalized oblique derivative boundary condition, Appl. Numer. Math. 108 (2016) $226-241$. 